\title{
Chronic Pain After Spinal Cord Injury: An Expedient Diagnostic Approach
}

\author{
J. H. Frisbie, MD, E. J. Aguilera, MD \\ Spinal Cord Injury Service of the Veterans Administration Medical Center, Brockton, \\ MA., USA.
}

\section{Summary}

The reliability of distinguishing central, musculoskeletal, and syringomyelic pain by two points of history: (1) pain quality and (2) pain location relative to the level of paralysis in spinal cord injury patients was tested by (1) physical examination, and (2) by radiographic imaging.

Fifty five incidents of chronic pain (median duration 10 years, range 3 weeks-42 years) were found in a survey of 66 spinal cord injured patients.

Central pain was suggested in 24 patients on the basis of a predominant 'neurogenic' pain quality: burning, stabbing, needles and pins, or numbness; and a location at or distal to the level of paralysis. Neurogenic pain was not associated with structural pathology in these patients.

Musculoskeletal pain was suggested in 20 instances on the basis of predominantly aching pain and a location at or distal to the level of paralysis. Aching pain was associated with degenerative joint disease (11 each); scoliosis, shoulder dislocation, contractures (2 each); fracture, soft tissue calcium deposit (1 each) in 19 patients.

Syringomyelic pain was suggested in 11 instances solely on the basis of pain location above the level of paralysis. Magnetic resonance imaging revealed extensive syringomyelia in 8 patients.

It is proposed that the quality and location of chronic pain can quickly suggest confirmatory examinations, sometimes revealing correctable causes.

Key Words: Pain; Spinal cord injury; Syringomyelia; Pain intractable; Pain measurement.

The complaints of chronic pain among spinal cord injury patients have been reported in papers on the subject over a period of many years (Riddoch, 1917; Holmes, 1919; Pollock et al., 1951; Nepomuceno et al., 1979; Woolsey, 1986). This pain has been described as 'phantom', referring to that which seems to emanate from areas of anaesthesia, or 'central', referring more specifically to that 
originating from the injured spinal cord itself (Munro, 1950; Guttmann, 1976; Melzack, 1978).

Beyond this explanation, other causes of pain, such as joint disease and syringomyelia (Rossier et al., 1985), are being recognised. In spite of this awareness, however, assessment of pain is often hampered for want of a convenient, reliable approach.

We have tested a convenient approach based on major features previously reported-quality and location of pain. The reliability of this approach in classifying causes of chronic pain has been tested by physical and radiographic examinations.

\section{Methods}

\section{Subjects}

Of the 73 patients assigned to the spinal cord injury service for long term care (inpatient or outpatient), 66 were qualified for interview. These patients had (1) intactness of memory for events from a time within the first 3 months of paralysis, and (2) a traumatic basis of paralysis.

Of those 66 patients 48 had experienced chronic pain, defined as an unpleasant sensation persisting longer than 3 weeks. The median age of these subjects was 55 years, range 33 to 89 ; the median duration of paralysis was 16 years, range 3 to 45 .

\section{Pain Description}

A standardised interview was conducted to describe chronic pain by its quality and location.

The quality of pain was divided into two categories-(1) 'neurogenic' pain, which consisted of (a) burning, (b) stabbing, (c) needles and pins, or (d) numbness, and (2) 'musculoskeletal', which consisted of aching pain. When the patient offered more than one description, the predominant complaint was recorded.

The location of pain was divided into two categories-(1) at or below the level of paralysis and (2) above the level of paralysis.

In addition, date of onset of pain relative to paralysis, associated spasms, intensity, consistency, course, and adverse and beneficial factors were determined.

\section{Pain Diagnosis}

Central pain was diagnosed from interview if (1) the quality of the pain was 'neurogenic' and if (2) the location of pain was at or distal to the level of paralysis. Central pain was confirmed if coinciding structural pathology, as determined by physical examination or radiographic reports, could not be found.

Musculoskeletal pain was diagnosed from interview if (1) the quality of pain was aching and if (2) the location of pain was at or distal to the level of injury. Musculoskeletal pain was confirmed if contracture, dislocation, fracture, trigger point, or degenerative joint disease corresponding to the location of pain could be ruled in by physical or radiographic examination.

Syringomyelic pain was diagnosed from interview if pain was located proximal 
to the level of paralysis. The quality of pain was disregarded when pain was so located. The diagnosis of syringomyelia was supported by an ascent of motor or sensory level on physical examination and confirmed by the demonstration of syringomyelia on magnetic resonant imaging.

Intensity of pain of any origin was graded as severe if pain required medication or interfered with daily activities, moderate if these conditions did not apply, or mild if the patient described his discomfort as such.

\section{Results}

The reliability of pain classification as described is indicated in Table I.

Table I Reliability of pain diagnosis by history alone

\begin{tabular}{lccc}
\hline Pain class & Diagnosed & Confirmed & Reliability \\
\hdashline Central & 24 & 24 & $100 \%$ \\
Musculoskeletal & 20 & 19 & $95 \%$ \\
Syringomyelic & 11 & 8 & $73 \%$ \\
A total of 55 instances of pain were classified for the 48 patients-pains \\
of different sources, location, or onset being reported in 7 patients.
\end{tabular}

The sensory losses in these pain patients are summarised in Table II.

Table II Completeness of sensory deficit vs cause of pain

\begin{tabular}{lrrrr}
\hline & \multicolumn{3}{c}{ Lesions } & Total \\
Pain class $^{\star}$ & & Complete & Incomplete & patients \\
Central & - & - & 16 & 24 \\
Musculoskeletal & 11 & 8 & 19 \\
Syringomyelic & 7 & 1 & 8 \\
Totals & 26 & 25 & 51 \\
\hline
\end{tabular}

The 4 instances of pain classification which were not confirmed were not included. Loss of sensation at the time of pain diagnosis is reported.

\section{Central pain}

The 'neurogenic' quality of pain was burning in 11 , stabbing in 7 , needles and pins in 4 , numbness in 2.

The location of pain was only at the level of paralysis in 10 subjects (bilateral in 7) and below the level of paralysis in 14 (multiple levels in 8). In 1 patient with a T6 level of paralysis and a mid-thoracic spine fracture, discomfort in the hands was reported. Although sensation was normal in the cervical dermatomes, radiographs revealed mid-cervical spine fractures. Thus we judged the location of pain in the hands to be at the level of spinal cord injury in this patient.

The median onset of central pain was 3 months after paralysis, with a range of 1 day to 16 months.

Flexor spasms were reported by 9 patients. On those occasions, occurring a few to innumerable times a day, paroxysms of pain associated with spasms caused patients to stop all voluntary activity. These spasms were often described as 
squeezing or pressing and were sometimes associated with sweating.

The intensity of central pain was severe in 15 (requiring medication or change in life style), moderate in 2 , and mild in 6 .

The course of central pain was characterised by complete or partial remission in 7 patients ( 3 weeks to 2 years after paralysis). Exacerbation of pain was reported in 6, explainable by the onset of complications (renal stones, fracture of lower extremities) in 3.

\section{Musculoskeletal pain}

By definition, the principal quality of pain was aching in all instances.

Also by definition, the location of pain was at or below the level of injury. Confirmation of musculoskeletal pain was possible in 19 of the 20 instances with evidence for degenerative joint disease (11 each), scoliosis, shoulder dislocation, contractures ( 2 each), and fracture, soft tissue calcium deposit (1 each). Pathology was most often found in the spine ( 8 instances).

The median onset of musculoskeletal pain was 8 years after spinal cord injury, with a range of 0.2 to 42 years. Six patients reported onset of chronic pain within 1 year of spinal cord injury, but 2 of these had similar complaints prior to spinal cord injury.

The intensity of pain was severe in 13 patients with treatment consisting of analgesics, local heat, tranquillizers, trigger point injection with local anaesthetics, and transcutaneous electrical nerve stimulation. Relief was incomplete.

Pain remission was achieved in 3 patients-in 2 by passive exercise to contractures, and in 1 by replacement of a degenerative shoulder joint with a prosthesis.

\section{Syringomyelia}

Eleven patients were believed to have post traumatic syringomyelia solely on the basis of interview.

Pain proximal to the level of paralysis, the only criterion for syringomyelia by history, was found at the neck in 7 , the shoulders in 2 , the arm in 1 , the trunk in 1. Pain began below the level of paralysis and extended above the level to the neck in 3 of these patients.

Eight of these patients had magnetic resonant imaging (MRI), and all had confirmation of syringomyelia. The syrinx extended from the site of original injury to the base of the medulla oblongata in 7 instances. The syrinx in several patients extended distal to the site of injury as well, reaching the conus medullaris in 1 .

Three patients did not have MRI. Two patients, paraplegic, had degenerative joint disease of the shoulder by X-ray examination which explained the pain complaint, located in the shoulder, and represented, in fact, musculoskeletal pain. One patient, quadriplegic, had chronic neck pain but declined the MRI and remained undiagnosed.

The predominant quality of the pain in the subjects proven to have syringomyelia was aching in 5 and burning or stabbing in 3 subjects.

The onset of pain ranged from 1 month to 24 years after paralysis, being less than a year in 4 patients. The onset of pain preceded the onset of ascending sensory 
loss or weakness in 5 patients. The interval between the onset of pain and the development of neurological signs ranged from 1 to 16 years.

Intensity of pain was rated as severe in 4 . Pain was constant and unremitting in all patients.

Muscle tenderness was sometimes found on physical examination with or without neurological change. A flexed neck posture, reduced stamina of neck muscles, and denervation of neck musculature were associated with high cervical syringomyelia in 3 of these patients (Frisbie et al., 1989).

\section{Discussion}

The reliability of pain classification by history alone was acceptable, in our judgment, as a guide to further diagnostic procedures.

We found it interesting that the quality of central pain-burning, stabbing, pins and needles, or numbness-but seldom aching (Davis and Martin, 1947; Pollock et al., 1951; Weinstein, 1962; Melzack and Loeser, 1978; Nepomuceno et al., 1979) — was similar to that of other nervous system trauma. Examples are the radiculopathies, the compression neuropathies as in carpal tunnel syndrome, ulnar palsy, meralgia paraesthetica (Adams and Victor, 1985; Schaumburg, 1988) or the gunshot wounds to the peripheral nerves resulting in causalgia (Mitchell, 1872).

The location of central pain at or below the level of paralysis has also been well described (Davis and Martin, 1947; Melzack and Loeser, 1978; Nepomuceno et al., 1979). Radiculopathy could conceivably account for some instances of 'central' pain because of its location at the level of spinal injury. For most subjects, however, with pain below the level of paralysis, one is left with the possibility that myelopathy itself generates pain, a proposed mechanism having been discussed (Melzack and Loeser, 1978).

One might ask why the early onset of central pain was not used as a criterion since this is very much a part of the central pain syndrome. Davis and Martin (1947) dated the onset as days to months after paralysis and Nepomuceno et al. (1979) found the onset less than 6 months after injury in $65 \%$ of subjects. We found, however, that the timing of onset did not distinguish central pain from pain of other sources.

The distinction of musculoskeletal from central pain based on pain quality has been suggested by Waisbrod (1984). Of 25 spinal cord injury patients with chronic pain a 'dragging' sensation was noted in 13 with 'lesions' whereas a burning sensation was complained of in 12 without lesions.

One might ask whether the aching of musculoskeletal pathology described here is to be believed in view of the fact that many of the patients had complete paralysis. However, complete transection of the cord in spinal cord injury is rare (Wozniewicz et al., 1983) and anaesthesia even by neurological examination is not absolute.

The distinction of the pain of syringomyelia from central or musculoskeletal pain, on the other hand, depends on the course of post traumatic syringomyelia, a cystic degeneration which begins at the site of injury and dissects. Although pain can begin below the level of paralysis, it is the ascent of pain above the level of paralysis that makes the diagnosis both a clear and a practical one. Pain is not only the most common presenting complaint of post traumatic syringomyelia but 
is the symptom most likely to be corrected by surgical drainage of the cyst (Faulhauer and Loew, 1978; Rossier et al., 1985).

One might question the high incidence of syringomyelia, $12 \%$ of those surveyed. Recently, however, an MRI survey of a similar population found a $22 \%$ prevalence of similarly sized cysts, $>50 \mathrm{~mm}$ in length (Hussey, 1989). Many more asymptomatic, smaller cysts were found in that survey and were undoubtedly screened out in this survey.

In summary, it is suggested that a description of chronic pain by quality and location can conveniently sort out central, musculoskeletal or syringomyelic pain. Confirmatory physical and radiographic examinations can then lead to the demonstration of structural pathology peripheral to or even within the spinal cord, some of which is correctable.

\section{References}

Adams RD, Victor M 1985 Pain in the back, neck, and extremities. In: Principles of Neurology, Ch. 10, 3rd edn. McGraw Hill Inc., New York, p 152.

Davis L, MARTIN J 1947 Studies upon spinal cord injuries. Fournal of Neurosurgery 4:483-491.

FAUlHAUER K, LOEW K 1978 The surgical treatment of syringomyelia, long term results. Acta Neurochirurgica 44:215-222.

Frisbie JH, Aguiler4 EJ, Foo D 1989 Flexed neck posture due to cervical post traumatic syringomyelia. Fournal of the American Paraplegia Society 12:3-5.

GutTman L 1976 Disturbances of sensibility. In: Guttmann L (ed): Spinal Cord Injuries. 2nd edn. Blackwell Scientific Publications, London, p 283.

Holmes C 1919 Pain of central origin. In: Contributions of Medical and Biological Research. PB Hoeber, New York, pp 235-246.

Hussey RW, Ha CY, Vijay M, Lipper M, Kubota R 1989 Prospective study of the occurrence rate of post-traumatic cystic degeneration of the spinal cord utilizing magnetic resonance imaging. The 35th Annual Conference of the Amercian Paraplegia Society, Abstract 41.

Kaplan LI, Grynbaum BB, LLOYd KE, RUSK HA 1962 Pain and spasticity in patients with spinal cord dysfunction. FAMA 182:918-925.

MELZACK R, LOESER JD 1978 Phantom body pain in paraplegics: Evidence for a central 'pattern generating mechanism' for pain. Pain 4:195-210.

Mitchell SW 1872 Injuries of Nerves and Their Consequence. Lippincott \& Co., Philadelphia

MunRo D 1950 Two year end results in the total rehabilitation of veterans with spinal cord and cauda equina injuries. New England Fournal of Medicine 242:1-16.

NePOMUCENo C, FINE PR, RICHARDS JS et al. 1979 Pain in patients with spinal cord injury. Archives of Physical Medicine and Rehabilitation 60:605-609.

Pollock L, Brown M, Boshes B et al. 1951 Pain below the level of injury of the spinal cord. Archives of Neurology and Psychiatry 65:319-322.

RiDDOCH G 1917 The reflex functions of the completely divided spinal cord in man, compared with those associated with less severe lesions. Brain 40:324-327.

Rossier AB, Foo D, Shillito J, Dyro FM 1985 Posttraumatic cervical syringomyella. Brain 108:439-461.

SCHAUmbURG HH 1988 Diseases of the peripheral nervous system. In WyNGaARDEN JF, SMITH LH (eds) Cecil Textbook of Medicine, 18th edn. WB Saunders Co, Philadelphia, pp 2267-2268

WAISBROD J 1984 Chronic pain in paraplegia. Neurosurgery 15:933-934.

WeinsteIn S 1962 Phantoms in paraplegia. Proceedings of the Veterans Administration Annual Conference 11:138-152.

WOOLSEY RM 1986 Chronic pain following spinal cord injury. Fournal of the American Paraplegia Society 9:39-41.

Wozniewicz B, Filipowicz K, Swiderska SK et al. 1983 Pathophysiological mechanism of traumatic cavitation of the spinal cord. Paraplegia 21: 312-317. 